\title{
A CLASS OF NULL SETS ASSOCLATED WITH CONVEX FUNCTIONS ON BANACH SPACES
}

\author{
JOHN RAINWATER
}

\begin{abstract}
A generalisation of the notion of "sets of measure zero" for arbitrary Banach spaces is defined so that continuous convex functions are automatically Gateaux differentiable "almost everywhere". It is then shown that this class of sets satisfies all the properties that one expects of sets of measure zero. Moreover (in a certain large class of Banach spaces, at least) nonempty open sets are not of "measure zero".
\end{abstract}

There are several different substitutes for sets of measure zero in infinite dimensional spaces (where $\sigma$-finite translation invariant measures do not exist); see, for instance, $[1,3,5,6]$. These have all been defined in separable Banach spaces and have generally been motivated by theorems which assert that locally Lipschitzian maps are Gateaux differentiable "almost everywhere", where the latter means "outside of a null set". In what follows we define a new class of null sets in arbitrary Banach spaces, with the motivation being Gateaux differentiability of convex functions.

DEFINITIONS: Let $f$ be a real-valued continuous convex function defined on a real Banach space $E$. We denote by $\mathcal{N}(f)$ the set of all points $x \in E$ where $f$ fails to be Gateaux differentiable. That is, for some $y \in E$, the limit

$$
\lim _{t \rightarrow 0} t^{-1}[f(x+t y)-f(x)]
$$

fails to exist.

By a null set we mean any subset of $\mathcal{N}(f)$, for some $f$ as above.

This definition changes the focus of attention from functions to sets: rather than attempting to show that convex continuous (hence locally Lipschitzian) functions are Gateaux differentiable almost everywhere (with respect to some given class of null sets), this property is guaranteed by the very definition of "null set". Of course, it then becomes important to show that this class of sets behaves the way that null sets should

Received 30 November 1989

The author wishes to thank David Preiss and Robert Phelps for conversations on the subject matter of this note.

Copyright Clearance Centre, Inc. Serial-fee code: 0004-9729/90 SA2.00+0.00. 
behave and that they are sets of Lebesgue outer measure zero in finite dimensional spaces. Some aspects of their behaviour are immediate from the definition:

(i) A subset of a null set is a null set, hence the intersection of any family of null sets is a null set.

(ii) Any translate of a null set is a null set.

(iii) Null sets are preserved under linear isomorphisms of the space.

(iv) A singleton $\left\{x_{0}\right\}$ is a null set. [The function $f(x)=\left\|x-x_{0}\right\|$ is not Gateaux differentiable at $x_{0}$.]

(v) Closed hyperplanes are null sets. [Let $f(x)=\left|\left\langle x^{*}, x\right\rangle\right|, x \in E$, where $x^{*} \in E^{*}, x^{*} \neq 0$.]

More generally,

(vi) The boundary of any closed convex set $C$ with nonempty interior is a null set. [If $0 \in \operatorname{int} C$, let $\mu$ be the Minkowski functional for $C$ (so that bdry $C=\{x: \mu(x)=1\}$ ) and let $f(x)=\max \{\mu(x), 1\}$, so that bdry $C \subset \mathcal{N}(f)$.]

Null subsets of separable Banach spaces have been characterised by Zajicek [10] (although he did not call them that); more about this later.

REMARK. Since adding a constant or a linear functional to a continuous convex function does not affect the points where the latter is Gateaux differentiable, for any $x_{0} \in E$ and $x^{*} \in \partial f\left(x_{0}\right)$ (the subdifferential of $f$ at $x_{0}$ ), we have $\mathcal{N}(f)=\mathcal{N}(g)$, where $g=f-x^{*}-f\left(x_{0}\right)-\left\langle x^{*}, x_{0}\right\rangle$. By definition, $\left\langle x^{*}, y-x_{0}\right\rangle \leqslant f(y)-f\left(x_{0}\right)$ for all $y \in E$, so the function $g$ is nonnegative; this means that in considering $\mathcal{N}(f)$, we can always assume that $f \geqslant 0$ on $E$ and, if we wish, that $f\left(x_{0}\right)=0$ for some predetermined point $x_{0}$.

The following basic property is less obvious.

Proposition 1. A countable union of null sets is a null set.

The proof requires the following lemmas.

Lemma 1. Suppose that $\left\{f_{n}\right\}$ is a sequence of nonnegative continuous convex functions on $E$ such that, for some $x_{0}$ and each $n=1,2,3, \ldots$, there exists $M_{n}>0$ such that $f_{n}(x) \leqslant M_{n} \cdot\left\|x_{0}-x\right\|$ for all $x \in E$. Then there exists a continuous convex function $f$ on $E$ such that $\bigcup \mathcal{N}\left(f_{n}\right)=\mathcal{N}(f)$.

Proof: Define $f$ by

$$
f(x)=\sum 2^{-n} M_{n}^{-1} f_{n}(x), \quad x \in E .
$$

It is clear that, as the supremum of a sequence (the partial sums) of nonnegative continuous convex functions, $f$ is convex, nonnegative and lower semicontinuous. Moreover, 
it is dominated by $\left\|x_{0}-x\right\|$, hence is necessarily continuous. Furthermore, if for some $k \geqslant 1$ the function $f_{k}$ fails to be Gateaux differentiable at a point $y$, then $f$ also fails to be differentiable at $y$. To see this, let $g_{k}=\sum_{n \neq k} 2^{-n} M_{n}^{-1} f_{n} ;$ then $g_{k}$ is continuous and convex and $f=f_{k}+g_{k}$. It is easily verified that since $\partial f_{k}(y)$ contains more than one element, the same is true of $\partial f(y)$. (More precisely, $\partial f_{k}(y)+\partial g_{k}(y) \subset \partial f(y)$; in fact, equality holds: see, for instance, $\left[7\right.$, p.54].) Thus, we have shown that $\bigcup \mathcal{N}\left(f_{k}\right) \subset \mathcal{N}(f)$. The reverse inclusion (which won't be needed in what follows) uses straightforward arguments to show that if $x_{n}^{*}$ is the Gateaux derivative of $f_{n}$ at $x, n=1,2,3, \ldots$, then $\left\|x_{n}^{*}\right\| \leqslant M_{n}$ and $x^{*}=\sum 2^{-n} M_{n}^{-1} x_{n}^{*}$ is the Gateaux derivative of $f$ at $x$.

We also need the following elementary lemma, which sharpens the standard result that a continuous convex function is locally Lipschitzian.

LEMMA 2. Suppose that $g$ is a nonnegative convex continuous function defined on a nonempty open convex set $D$. Then

$$
D=\bigcup\left\{D_{m, n}: n, m=1,2,3, \ldots\right\}
$$

where each set $D_{m, n}$ is convex and open and the restriction of $g$ to $D_{m, n}$ has Lipschitz constant $m n$.

Proof: First, for $m=1,2,3, \ldots$, let $D_{m}=\{x \in D: g(x)<m\}$ and let

$$
D_{m, n}=\left\{x \in D_{m}: \operatorname{dist}\left(x, E \backslash D_{m}\right)>1 / n\right\}
$$

These sets are open and convex (some may be empty) and $D=\bigcup_{m} D_{m}$. Since $D_{m}=$ $\bigcup_{n} D_{m, n}$, we have $D=\bigcup_{m, n} D_{m, n}$. Suppose, now, that $x$ and $y$ are distinct points in $D_{m, n}$. Let $\alpha=\|x-y\|>0$ and consider

$$
z=y+(y-x) / n \alpha .
$$

We cannot have $z \in E \backslash D_{m}$ because $\|z-y\|=1 / n$ and $y \in D_{m, n}$. It follows that $g(z)<m$ and, since

we have

$$
y=(n \alpha z+x) /(1+n \alpha),
$$

so that

$$
g(y)-g(x) \leqslant n \alpha[g(z)-g(x)] /(1+n \alpha) \leqslant n \alpha[g(z)-g(x)]<m n \alpha=m n \cdot\|y-x\| .
$$

Repeating the foregoing argument with $x$ and $y$ interchanged shows that

$$
|g(y)-g(x)| \leqslant m n \cdot\|y-x\|,
$$


which was to be proved.

(The nonnegativity hypothesis may be dropped; one ends up with $2 m n$ in place of $m n$.)

Proof of Proposition 1: It suffices to prove that if $A$ is a null set, then there exists a sequence of nonnegative functions $\left\{f_{n}\right\}$ as in Lemma 1 such that $A \subset \bigcup \mathcal{N}\left(f_{n}\right)$. [This will prove the proposition, of course, since a countable union of null sets will thus be contained in a countable union $U$ of sets, each of the form $\bigcup \mathcal{N}\left(f_{n}\right)$, so $U$ is again a set of the same form. By Lemma $1, U$ is contained in $\mathcal{N}(f)$ for some $f$.] Now, by definition, there exists a convex continuous function $g$ (which we may assume to be nonnegative) such that $A \subset \mathcal{N}(g)$. For later use, we assume that $g$ is defined on some nonempty open convex subset $D$, not necessarily on all of $E$, and that $\mathcal{N}(g) \subset D$ is defined in the obvious way. Write $D=\bigcup D_{m, n}$ as in Lemma 2. Fix $x_{0} \in D$ and assume, without loss of generality, that $g\left(x_{0}\right)=0$. For positive integers $m$ and $n$, let $f_{m, n}$ denote the inf-convolution of $g$ and $n m\|\cdot \cdot\|$; that is

$$
f_{m, n}(x)=\inf \{g(y)+m n\|y-x\|: y \in D\}, \quad x \in E
$$

As is well known (see, for instance, [7, p.33]), $f_{m, n}$ is a convex Lipschitzian function on $E$, with Lipschitz constant $m n$, such that $f_{m, n}(x)=g(x)$ for $x \in D_{m, n}$. Moreover, since $g \geqslant 0$ and $g\left(x_{0}\right)=0$, the definition implies that $0 \leqslant f_{m, n}(x) \leqslant m n \cdot\left\|x-x_{0}\right\|$ for all $x$. If $g$ fails to be differentiable at a point $x \in D$, then $x \in D_{m, n}$ for some $m, n$ and hence $f_{m, n}$ fails to be differentiable at $x$. Thus, $\mathcal{N}(g) \subset \bigcup \mathcal{N}\left(f_{m, n}\right)$ and the proof is complete.

REMARK. Our definition of "null set" is equivalent to saying that any convex continuous function on all of $E$ is Gateaux differentiable outside of a null set. We would like this latter assertion to be true for any convex continuous function $g$ defined on an arbitrary nonempty open convex subset $D$ of $E$. This follows from the last part of the proof of Proposition 1 , since $\mathcal{N}(g) \subset \bigcup \mathcal{N}\left(f_{m, n}\right)$ where each $f_{m, n}$ is defined on all of $E$ (and $\bigcup \mathcal{N}\left(f_{m, n}\right)$ is a null set $)$.

Proposition 1 has an immediate corollary (which can be proved directly by looking at functions of the form $\left.f(x)=\sum 2^{-n}\left(1+\left\|x_{n}\right\|\right)^{-1} \cdot\left\|x-x_{n}\right\|\right)$.

(vii) Countable sets are null sets. (In the real line, the converse is true: null sets are at most countable. See, for instance, [7, p.10]).

Zajíček has very nicely characterised the null subsets of a separable real Banach space $E$ in the following terms: A subset $M$ of $E$ is called a $(c-c)$-hypersurface if - roughly - it is the graph in $E$ of a function which is the difference of two convex Lipschitzian functions. More precisely, it is required that there exist a closed hyperplane 
$H$ in $E$, a vector $v$ in $E$ such that $E=H+\mathbf{R} v$, and two convex Lipschitzian functions $f, g$ defined on $H$ such that

$$
M=\{x+[f(x)-g(x)] v: x \in H\}
$$

Theorem 2 of [10] asserts that a subset of $E$ is a null set if and only if it is contained in the union of countably many $(c-c)$-hypersurfaces. [By using the method of proof of Proposition 1, it can be shown that the graph of the difference of two convex continuous functions is contained in a countable union of graphs of convex Lipschitzian functions, so that Zajićek's theorem is equivalent to the formally weaker result in which the word "Lipschitzian" is deleted.] Note that Zajícek's theorem immediately yields Proposition 1 for separable spaces.

An important property of any class of null sets is that nonempty open sets not be null sets. This is not always the case; for instance, in the spaces $\ell^{1}(\Gamma)$ ( $\Gamma$ uncountable) and $L^{\infty}[0,1]$ the norms are nowhere Gateaux differentiable $[7, \mathrm{pp} .3,70]$, so these spaces are themselves null sets. The same is true of the space $\ell^{\infty}$, since it admits a nowhere Gateaux differentiable seminorm $[7, p .13]$. It is not too difficult to show that the norm in $\ell^{\infty}(\Gamma)^{*}$ ( $\Gamma$ infinite) is also nowhere Gateaux differentiable.

Definition: A Banach space $E$ is said to be a Gateaux differentiability space (GDS) provided every convex continuous function $f$ defined on an open convex subset $D$ of $E$ is Gateaux differentiable at the points of a dense subset of $D$. We say that $E$ is a weak Asplund space if the set of points of differentiability of $f$ contains a dense $G_{\delta}$ subset of $D$.

Separable Banach spaces, in fact, all weakly compactly generated Banach spaces, are weak Asplund spaces. It is an open question whether every GDS is a weak Asplund space. For further discussion and references, see, for instance, [4] and [7]. As noted above, the spaces $\ell^{1}(\Gamma)$ ( $\Gamma$ uncountable), $L^{\infty}[0,1], \ell^{\infty}$ and $\ell^{\infty}(\Gamma)^{*}(\Gamma$ infinite) are not even Gateaux differentiability spaces.

The following important property is now immediate from the definitions.

(viii) In a Gateaux differentiability space $E$, nonempty open sets are not null sets.

Stated in another form, in a GDS, the complement of a null set (called a "co-null set") is necessarily dense. Proposition 1 implies that co-null subsets of a GDS have a Baire-like property: A countable intersection of co-null sets is co-null, and is therefore dense.

By putting together some known results, we see easily that the relation between null sets and Gateaux differentiability spaces is quite close. 
Proposition 2. For a real Banach space $E$ the following assertions are equivalent.

(1) $E$ is not a GDS.

(2) There exists an equivalent norm on $E$ which is nowhere Gateaux differentiable.

(3) $E$ is a null set.

Proof: (1) implies (2). It is shown in [7, p.63] that being a GDS is equivalent to being an MDS (that is, every Minkowski functional on $E$ is Gateaux differentiable at the points of a dense set) and it was proved in [4, Proposition 5] that if $E$ is not an MDS, then it admits an equivalent norm which is nowhere Gateaux differentiable. It is obvious that (2) implies (3) and that (3) implies (1).

In order to compare the class of null sets with the classes which have been studied previously, we must look at separable Banach spaces and at those null sets which are also Borel sets (since the "exceptional sets" of [1], the Gaussian null sets of $[6]$ and the Haar null sets of [3] are all Borel sets). In a separable Banach space, Mazur's theorem (reformulated) asserts that each $\mathcal{N}(f)$ is a first category set, but in more general spaces little is known about their Borel type. Talagrand [8] has shown that in a certain $\ell^{1}(\Gamma)$ (namely, for $\Gamma$ the uncountable interval of ordinals $\left[0, \omega_{c}\left[\right.\right.$, where $\omega_{c}$ is the first ordinal having the power of the continuum), there exists a continuous convex function $f$ such that $\mathcal{N}(f)$ is nowhere dense but is not even Borel-universally measurable. Since $\ell^{1}(\Gamma)$ is not a GDS, the following question still remains: If $E$ is a GDS, are the sets $\mathcal{N}(f)$ necessarily Borelian? This question can be sidestepped, of course, by defining "null set" to be any Borel set which is contained in a set of the form $\mathcal{N}(f)$. All of the properites (i) - (viii) listed above hold for this definition, but a new question arises. If we want to say that every continuous convex function $f$ is Gateaux differentiable outside of a "null set", then given $f$ we must produce a Borel set $B$ and a continuous convex function $g$ such that $\mathcal{N}(f) \subset B \subset \mathcal{N}(g)$. This does not appear obvious.

It is easy to produce an example of a null set which is not of the form $\mathcal{N}(f)$ : in $R^{2}$, the real axis is a null set, so any subset of the $x$-axis is a null set, but it cannot be an $\mathcal{N}(f)$ unless it is an $F_{\sigma}$. We know of no characterisation of those subsets of the plane which have the form $\mathcal{N}(f)$.

As shown in [6], the class of Aronszajn exceptional sets is contained in the class of Gaussian null sets and the latter class is strictly contained in the class of Haar null sets. [It is open whether the Gaussian null sets coincide with Aronszajn's exceptional sets, although Bogachev [2] has shown that they are the same for the class of sets which are measurable relative to every densely differentiable measure on the space. (See [2] for the relevant definitions.)] It follows from Aronszajn's differentiability theorem for locally Lipschitzian mappings that for a continuous convex function $f$ on a separable Banach 
space, the $F_{\sigma}$ set $\mathcal{N}(f)$ is one of Aronszajn's exceptional sets. Since a Borel subset of an exceptional set is an exceptional set, we conclude that in a separable Banach space, any null set which is also a Borel set is an Aronszajn exceptional set. This inclusion is strict, since null sets in the real line are at most countable, while there exist Lipschitzian functions on the line which have an uncountable set of points of nondifferentiability. (In fact, any $G_{\delta \sigma}$ set of Lebesgue measure zero is such a set; see [9, Lemma 8].)

Using Rademacher's theorem, Aronszajn noted that in finite dimensional spaces, his exceptional sets coincide with the Borel sets of Lebesgue measure zero, hence in this case null sets all have Lebesgue outer measure zero. [This also follows from the much more easily proved fact (see [7, p.11], for instance) that continuous convex functions on finite dimensional spaces are differentiable almost everywhere.]

If null sets (or any of the other generalisations of sets of measure zero) were, in fact, the sets of measure zero for some $\sigma$-finite measure, then it would follow that any pairwise disjoint family of non-null sets would be at most countable. This fails, however, for all of the families mentioned above. A simple example for the Gaussian null sets (hence valid for null sets) is presented in [6]; it consists of an uncountable collection of pairwise disjoint compact subsets of $\ell^{2}$, each of which is not Gaussian null (hence not null).

A straightforward generalisation of the foregoing notion of null set is possible, using the following types of derivatives.

Definition: Let $\beta$ be any nonempty family of bounded subsets $S$ of the Banach space $E$ whose union is all of $E$. We say that a continuous convex function $f$ on a nonempty open convex subset $D$ of $E$ is $\beta$-differentiable at $x \in D$ provided that for each $S$ in $\beta$, the limit in (1) exists uniformly for $y \in S$. (This yields, among others, the Gateaux derivative if $\beta$ consists of all finite sets, the weak Hadamard derivative if $\beta$ consists of all weakly compact sets and the Fréchet derivative if $\beta$ consists of all bounded sets.)

We denote by $\mathcal{N}_{\beta}(f)$ the set of points in $D$ where $f$ fails to be $\beta$-differentiable, and call a subset of $E \beta$-null provided it is contained in $\mathcal{N}_{\beta}(f)$ for some continuous convex $f$. It is easily verified that all of the properties (i) - (vii) listed above, as well as Proposition 1 , go through for $\beta$-null sets. (In verifying Proposition 1 , one must check that if the sum of two convex continuous functions is $\beta$-differentiable, then so is each summand.) The reason for not concentrating on the more general notion is that the class of spaces for which nonempty open sets are not $\beta$-null sets can be somewhat restrictive. In the Fréchet case, for instance, this forces $E$ to be an Asplund space.

\section{REFERENCES}

[1] N. Aronszajn, 'Differentiability of Lipschitzian mappings between Banach spaces', Studia 
Math. 57 (1976), 147-190.

[2] V.I. Bogachev, 'Negligible sets in locally convex spaces', Math. Notes 36 (1984), 519-526.

[3] J.P.R. Christensen, Measure theoretic zero sets in infinite dimensional spaces and applications to differentiability of Lipschitz mappings, 2-ieme Coll. Anal. Fonct. (Bordeaux, 1973). Publ. du Dept. Math. Lyon t. 10-2 (1973), 29-39 .

[4] D.G. Larman and R.R. Phelps, 'Gateaux differentiability of convex functions on Banach spaces', J. Lond. Math. Soc. 20 (1979), 115-127.

[5] P. Mankiewicz, 'On the differentiability of Lipschitz mappings in Frechet spaces', Studia Math. 45 (1973), 15-29.

[6] R.R. Phelps, 'Gaussian null sets and differentiability of Lipschitz maps on Banach spaces', Pac. J. Math. 77 (1978), 523-531.

[7] R.R. Phelps, Convex Functions, Monotone Operators and Differentiability: Lect. Notes in Math. 1364 (Springer-Verlag, Berlin, Heidelberg, New York, 1989).

[8] M. Talagrand, 'Deux exemples de fonctions convexes', C.R. Acad. Sci. Paris 288 (1979), 461-464.

[9] Z. Zahorski, 'Sur l'ensemble des points de non-derivabilite d'une fonction continue', Bull. Soc. Math. France 74 (1946), 147-178.

[10] L. Zajiček, 'On the differentiation of convex functions in finite and infinite dimensional spaces', Czechoslovak Math. J. 29 (1979), 340-348.

Department of Mathematics GN-50

University of Washington

Seattle WA 98195

United States of America 